\title{
Factores asociados con el consumo diario de cigarrillo en adolescentes estudiantes de básica secundaria de Bucaramanga, Colombia
}

\author{
Jorge Arturo Martínez, Walter Amaya, Horacio Alfredo Campillo, \\ Adalberto Campo, Luis Alfonso Díaz \\ Grupo de Neuropsiquiatría, Facultad de Medicina, Universidad Autónoma de Bucaramanga, \\ Bucaramanga, Santander, Colombia.
}

Introducción. El consumo diario de cigarrillo se asocia con una alta morbimortalidad en la adultez. Este patrón de consumo se inicia habitualmente en la adolescencia temprana. En adolescentes, el consumo diario de cigarrillo es factor de predicción importante del uso de sustancias ilegales que pueden producir dependencia. Se desconoce la frecuencia de consumo diario de cigarrillo en estudiantes colombianos.

Objetivo. Determinar la prevalencia y los factores asociados con el consumo diario de cigarrillo en adolescentes estudiantes de sexto a noveno grado del área metropolitana de Bucaramanga, Colombia.

Materiales y métodos. Se realizó un estudio transversal. Una muestra probabilística de 2.586 estudiantes diligenció en forma anónima una encuesta que indagaba el consumo de sustancias legales e ilegales.

Resultados. La edad promedio fue 13,0 años; $54,9 \%$ era varones; $32,0 \%$ cursaba sexto grado; $31,7 \%$, séptimo; $25,2 \%$, octavo, y $11,1 \%$, noveno; $67,1 \%$ estudiaba en colegios oficiales. El consumo diario de cigarrillo durante el mes anterior fue de 3,8\% (IC95\% 2,7 a 4,9). La regresión logística mostró que tener un mejor amigo fumador o consumidor de alcohol (OR=3,71; IC95\% 1,71 a 8,04), referir mal rendimiento académico (OR=3,83; IC95\% 1,03 a 14,18) y tener más años de edad (OR=1,44; IC95\% 1,19 a 1,75) se asociaba con el consumo diario de cigarrillo. Conclusión. Uno de cada 26 estudiantes de básica secundaria del área metropolitana de Bucaramanga consume diariamente cigarrillo. Tener un mejor amigo fumador es el factor más fuertemente asociado.

Palabras clave: tabaquismo, epidemiología, prevalencia, adolescente, estudiantes, salud pública.

Social factors associated with the daily cigarette smoking among middle-school student adolescents in Bucaramanga, Colombia

Introduction. Daily cigarette smoking is associated with high morbidity and mortality in adults. This pattern of tobacco use is established during the early adolescence. Among adolescents, daily cigarette smoking is a strong predictor of illegal, addictive substance use. The prevalence of daily cigarette smoking is not known in Colombian adolescent middle-school students.

Objective. To assess smoking prevalence, daily cigarette smoking and associated factors among middle school-students in Bucaramanga, northeast Colombia.

Materials and methods. An anonymous questionnaire concerning illegal and legal substance use was administered to a random sample of 2,586 students.

Results. The group mean age was 13.0 years; $54.9 \%$ was male; $32.0 \%$ was in sixth grade, $31.7 \%$ in seventh, $25.2 \%$ in eighth and $11.1 \%$ in ninth grade, with $67.1 \%$ enrolled in public schools. The prevalence of daily cigarette smoking lasting the month prior to the questionnaire was $3.8 \%(95 \% \mathrm{Cl}$ 2.7-4.9). Logistic regression showed a significant association between being the best friend of a smoker or a drinker of alcohol (OR=3.71; 95\% $\mathrm{Cl} 1.71-8.04)$, having low academic performance perception $(\mathrm{OR}=3.83$; $95 \% \mathrm{Cl} 1.0-14.2)$, and being older age (OR=1.4; $95 \% \mathrm{Cl} 1.2-1.8)$.

Conclusions. These data demonstrated that one in 26 middle-school student adolescents from 
Bucaramanga is an everyday smoker. The most important associated factor was being the best friend of a smoker or a drinker of alcohol.

Key words: smoking, epidemiology, prevalence, adolescent, students, public health.

La adolescencia es la etapa con el mayor riesgo para el inicio del consumo de sustancias que pueden producir dependencia (1). El consumo diario de cigarrillo, generalmente, se inicia y establece en etapas tempranas de la adolescencia (2). El consumo diario de cigarrillo tiene complicaciones médicas, generalmente a largo plazo, y es responsable de un número importante de morbimortalidad prevenible en la vida adulta (3). Así mismo, el consumo diario de cigarrillo se asocia a corto plazo con otras conductas de riesgo para la salud física y mental en adolescentes (4$6)$.

La experimentación con tabaco, en la mayoría de los casos en forma de cigarrillo, es frecuente en estadios tempranos de la adolescencia, generalmente en edades comprendidas entre los 13 y 15 años (7). La mayoría de los adolescentes fuman ocasionalmente sin que ello esté relacionado con problemas psicosociales significativos (8). Sin embargo, un número importante desde la perspectiva de salud pública, consolida un patrón de consumo regular de cigarrillo $(2,9,10)$.

En estudiantes de media vocacional se ha observado que el consumo diario de cigarrillo se asocia en forma significativa con una serie de consecuencias negativas tales como consumo de otras sustancias, por ejemplo, alcohol y marihuana, deserción y fracaso académico, accidentes, violencia, relaciones sexuales de alto riesgo para embarazos no planeados 0 enfermedades transmisibles sexualmente, y suicidio (11-13).

El consumo diario de cigarrillo tiene repercusiones en lo económico, político y social, de tal suerte

\footnotetext{
Correspondencia:

Adalberto Campo, Facultad de Medicina, Universidad Autónoma de Bucaramanga, Calle 157 \# 19-55, Cañaveral Parque, Bucaramanga, Colombia. acampoar@unab.edu.co.
}

Recibido: 18/04/05; aceptado: 09/09/05 que ha sido motivo de interés y preocupación constante. En este contexto, los estudios epidemiológicos y la vigilancia constante del consumo diario de cigarrillo adquieren especial relevancia, puesto que permiten elaborar y evaluar políticas adecuadas y efectivas para la prevención e intervención temprana de esta problemática, así como para la previsión de la dimensión que pueda alcanzar en los próximos años. Hasta la fecha, este grupo poblacional había sido excluido en otras investigaciones realizadas en Bucaramanga, Colombia $(14,15)$.

El objetivo general de este estudio fue evaluar la prevalencia del consumo de sustancias en adolescentes de sexto a noveno grado (básica secundaria) del área metropolitana de Bucaramanga. En este informe se presentan la prevalencia de consumo diario de cigarrillo y algunos factores asociados de gran relevancia.

\section{Materiales y métodos}

El presente es un estudio poblacional de corte transversal evaluado y aprobado por el Comité de Ética de la Facultad de Medicina de la Universidad Autónoma de Bucaramanga en el marco del proceso de investigación formativa. Igualmente, el proyecto fue aprobado por las autoridades académicas de las instituciones educativas y los padres de familia. Finalmente, se solicitó el consentimiento de los estudiantes participantes después de explicar los objetivos del estudio, asegurar la confidencialidad y el mínimo riesgo de la participación, como lo exigen las leyes colombianas $(16,17)$.

La población objeto del estudio estuvo conformada por los 69.284 estudiantes matriculados durante 2004 en 191 centros de educación secundaria del área metropolitana de Bucaramanga, tanto de colegios públicos como privados. Se calculó una muestra de 1.274 estudiantes con el fin de detectar una prevalencia de consumo de marihuana de $3,5 \pm 1,0 \%$, con un nivel de confianza de $95 \%$. 
Se realizó un muestreo probabilístico por conglomerados, en el que cada salón de clase representaba un conglomerado. Se asumió que cada conglomerado estaba formado por 20 estudiantes, por lo que se estimó la existencia de 3.964 conglomerados potenciales en el total de instituciones, de donde se extrajo aleatoriamente los 64 conglomerados necesarios para completar una muestra adecuada. El total de conglomerados por encuestar en cada institución se definió por muestreo aleatorio simple al asignar a cada centro educativo un número de conglomerados potenciales igual a la veinteava parte del total de estudiantes matriculados. Una vez se definió el total de conglomerados a encuestar en cada centro, se seleccionaron por muestreo aleatorio simple tantos salones como fuera necesario a partir de la lista de cursos suministrada por los directivos. Así se buscaba garantizar la inclusión de los conglomerados de aquellos establecimientos educativos con menor número de estudiantes.

Se empleó un cuestionario autoadministrado, voluntario, confidencial y anónimo, que se entregó en sobre cerrado y se diligenció en el aula de clase bajo la instrucción y supervisión de uno de los investigadores. Su estructura se basó en los modelos del Sistema de Vigilancia Epidemiológica para el Consumo de Sustancias Psicoactivas en Estudiantes de Secundaria (VESPA) desarrollado por el Plan Municipal de Prevención de Medellín en $1994(18,19)$. Este formato hacía preguntas referentes a la frecuencia de consumo de sustancias legales (tabaco, alcohol, tranquilizantes, estimulantes, inhalantes y hongos) y sustancias ilegales (marihuana, cocaína, bazuco, opiáceos y LSD). Además, y siguiendo la misma metodología del sistema VESPA, se indagó sobre el uso de nuevas sustancias emergentes no incluidas en el sistema como el éxtasis.

El consumo de tabaco, a diferencia de lo propuesto por el sistema VESPA, se evaluó por medio de preguntas referentes al antecedente de consumo, el uso algún día durante el mes anterior y el uso diario durante el último mes. Este grupo se categorizó como fumador actual o con consumo diario de cigarrillo y, además, se le indagó sobre la cantidad de cigarrillos consumidos al día. Se tomó esta definición de fumador porque cumple con el primer criterio para dependencia de nicotina según los lineamientos vigentes de la Organización Mundial de la Salud y la Asociación Psiquiátrica Americana. Sólo una parte de los fumadores regulares reúnen criterios para dependencia de nicotina $(20,21)$. Igualmente, las investigaciones más recientes en adolescentes utilizan esta definición. Por otra parte, esta categorización posibilita mejores comparaciones con la población adulta. Finalmente, entre los fumadores, los consumidores diarios de cigarrillo representan el verdadero problema de salud pública, ya que tienen mayor frecuencia de dependencia de nicotina, más dificultades para abandonar el consumo y mayor riesgo de trastornos mentales y de enfermedad física (22).

La escala CAGE se utilizó para estimar el riesgo de alcoholismo o consumo abusivo de alcohol. Este instrumento toma su nombre del acrónimo en inglés derivado de las cuatro preguntas que lo componen, referentes a la crítica social $(C)$, la culpa individual (A), la ingestión matutina (G) y la necesidad sentida de dejar de consumir el alcohol (E). La respuesta afirmativa a tres o más indica un patrón de consumo abusivo de alcohol (23). Este instrumento de tamizaje ha mostrado en otros estudios una buena sensibilidad $(60 \%$ a $90 \%)$ y una especificidad aceptable (40\% a 60\%) (24). No obstante, para conocer la validez indirecta de esta escala dicotómica se determinó la consistencia interna en la muestra estudiada con la fórmula 20 de Kuder-Richardson (25), la cual es equivalente matemáticamente a la prueba de alfa de Cronbach usada en las escalas tipo Likert (26). Se acepta que la consistencia interna es una característica del patrón de respuesta de una escala en una población específica y no una propiedad de la escala, por lo que debe informarse cada vez que se utilice el instrumento (27).

Los datos se analizaron con STATA para Windows 9.0 (28). En el modelo descriptivo se calcularon las distribuciones de frecuencia de todas las variables, incluidos sus intervalos de confianza de $95 \%$ (IC95\%), ajustadas según las características del muestreo con las rutinas del módulo survey data del programa. En caso de hacerse comparaciones, este módulo utiliza la 
prueba $\chi^{2}$ modificada por Rao y Scott, en la cual se computa por medio de una corrección de segundo orden de la razón de verosimilitud (29). Así, se determinaron razones de disparidad (OR), que, aunque no son la medida de asociación que se recomienda para los estudios transversales, constituyen la mejor forma disponible para evaluar asociaciones en encuestas trasversales con muestreo complejo (30).

Para ajustar por variables de confusión, se realizó un análisis multivariado mediante un modelo de regresión logística, teniendo igualmente en cuenta el proceso de muestreo. Se incluyeron en este modelo las variables que en el análisis bivariado mostraron valores de probabilidad menores de 0,20 . El modelamiento se llevó a cabo siguiendo las recomendaciones de Greenland (31). Se calcularon OR con IC de $95 \%$. En este modelamiento se aceptaron como significativas diferencias con valores de probabilidad menores de 0,05 .

\section{Resultados}

Se encuestaron 64 conglomerados que agrupaban a 2.609 estudiantes. Esto se debió a que el promedio de estudiantes por salón fue de 40,8 (rango 24 a 47) estudiantes y no de 20 estudiantes como inicialmente se estimó. Un número de 6 $(0,22 \%)$ estudiantes no aceptaron participar en el estudio y $17(0,65 \%)$ cuestionarios se anularon por inconsistencias de información. Finalmente, se incluyeron en el análisis 2.586 encuestas.

La edad de la muestra estudiada, ajustada por el muestreo, osciló entre 10 y 19 años, con una media de 13,0 años (IC95\% 12,9 a 13,1). Teniendo en cuenta el efecto del muestreo, 54,9\% (IC95\% 51,2 a 56,7) de los participantes eran varones y $67,1 \%$ (IC95\% 66,4 a 67,8) estudiaban en colegios oficiales; 32,0\% (IC95\% 39,4 a 34,6) estaban en sexto grado, $31,7 \%$ en séptimo (IC95\% 29,0 a $34,4), 25,2 \%$ (IC95\% 22,9 a 27,6) en octavo y $11,1 \%$ (IC95\% 9,9 a 12,3) en noveno; $6,2 \%$ (IC95\% 4,9 a 7,6) residían en estrato 1, 15,3\% (IC95\% 13,2 a 17,3) en el 2, 29,0\% (IC95\% 26,4 a 31,5 ) en el 3, 21,6\% (IC95\% 19,4 a 23,8) en el $4,10,4 \%$ (IC95\% 8,8 a 11,9) en el 5, 10,3\% (IC95\% 8,9 a 11,6) en el 6 y 7,3\% (IC95\% 5,8 a $8,7)$ omitieron esta información.
En relación con el estado de salud durante el mes anterior al estudio, 47,7\% (IC95\% 45,0 a 50,4) lo informó como excelente, 35,4\% (IC95\% 32,8 a 38,0 ) como bueno, 13,9\% (IC95\% 12,0 a 15,8) como regular y 3,0\% (IC95\% 2,1 a 4,0) como malo. En cuanto al rendimiento académico, $26,4 \%$ (IC95\% 24,0 a 28,8) lo consideraban excelente, 40,6\% (IC95\% 38,0 a 43,3), bueno, 26,1\% (IC95\% $23,7$ a 28,6$)$, regular y $6,8 \%$ (IC95\% 5,4 a 8,3 ) malo.

Respecto al consumo de cigarrillo, se encontró que la prevalencia ajustada por el muestreo fue de $16,7 \%$ (IC95\% 15,8 a 19,9) para haber fumado alguna vez en la vida, $11,1 \%$ (IC95\% 8,8 a 13,3) para haber fumado algún día durante el mes anterior, y de 3,8\% (IC95\% 2,7 a 4,9) para haber fumado todos los días durante el último mes (consumo diario de cigarrillo). La cantidad de cigarrillos fumados por día osciló entre uno y cuarenta, con promedio ajustado por el muestreo de 11,9 (IC95\% 11,6 a 12,1). Por su lado, 21,3\% (IC95\% 19,0 a 23,6) de los estudiantes manifestaron haber consumido alcohol durante el último mes, mientras que la prevalencia ajustada de consumo de alcohol sugestivo de alcoholismo fue de 10,7\% (IC95\% 9,0 a 12,4). La consistencia interna de la escala CAGE medida con la fórmula 20 de Kuder-Richardson fue 0,655. Por su parte, $68,2 \%$ (IC95\% 65,7 a 70.8) informó consumo de alcohol o cigarrillo por parte de un hermano u otro familiar, mientras que $31,8 \%$ (IC95\% 30,1 a 34,6) manifestó que el mejor amigo consumía alcohol o cigarrillo.

En el análisis bivariado concerniente a la edad se observó que los estudiantes con consumo diario de cigarrillo tenían una edad promedio de 14,1 (IC95\% 13,7 a 14,6) comparado con 12,9 (IC95\% 12,8 a 13,0); esta diferencia fue estadísticamente significativa $(p=0,0001)$. Los varones presentaban consumo diario de cigarrillo en 5,4\% (IC95\% 3,8 a 7,1 ) versus $1,6 \%$ (IC95\% 0,7 a 2,6) informado por las mujeres, diferencia también significativa (OR=1,97 IC95\% 1,09 a 3,58). La prevalencia ajustada de consumo diario de cigarrillo por escolaridad fue la siguiente: en sexto grado de $1,0 \%$ (IC95\% 0,3 a 1,7), 4,8\% (IC95\% 2,6 a 7,1) en séptimo, 4,7\% (IC95\% 2,2 a 7,2) en octavo y $5,9 \%$ (IC95\% 3,3 a 8,5) en noveno, diferencia que 
Cuadro 1. Consumo diario de cigarrillo según el grado de escolaridad, la percepción del estado de salud y el rendimiento académico en adolescentes estudiantes de básica secundaria de Bucaramanga, Colombia.

\begin{tabular}{lccc}
\hline Variable & OR & IC 95\% & $\boldsymbol{p}$ \\
\hline Grado & & & \\
Sexto & 1,00 & - & - \\
Séptimo & 3,28 & $1,15-9,38$ & 0,027 \\
Octavo & 3,40 & $1,21-9,62$ & 0,021 \\
Noveno & 4,47 & $1,62-12,35$ & 0,005 \\
Percepción del estado & & & \\
de salud & & & \\
Excelente & 1,00 & - & - \\
Bueno & 1,41 & $0,65-3,12$ & 0,374 \\
Regular & 2,09 & $0,82-5,30$ & 0,118 \\
Malo & 5,56 & $1,82-16,98$ & 0,003 \\
Percepción de rendimiento & & & \\
académico & & & \\
Excelente & 1,00 & - & - \\
Bueno & 1,83 & $0,62-5,41$ & 0,268 \\
Regular & 2,01 & $0,75-5,37$ & 0,159 \\
Malo & 5,90 & $1,66-20,97$ & 0,007 \\
\hline
\end{tabular}

fue estadísticamente significativa $(p=0,012)$; los OR para consumo diario de cigarrillo según el grado de escolaridad, tomando como referencia los estudiantes de sexto grado, se observan en el cuadro 1.

Según el estado de salud, 2,7\% (IC95\% 1,3 a 4,1 ) estudiantes que consideraban su salud excelente informaron consumo diario de cigarrillo, mientras que así lo informaban 3,2\% (IC95\% 1,9 a 4,5 ) de quienes referían buena salud; $5,6 \%$ (IC95\% 2,4 a 8,7) de los que informaban estado de salud regular, y $16,8 \%$ (IC95\% 4,6 a 28,9) de los que se percibían con mala salud. Estas diferencias alcanzaron significación estadística $(p<0,001)$; los OR según la percepción del estado de salud, tomando como referencia el estado de salud informado como excelente, aparecen en el cuadro 1.

En lo relacionado con el rendimiento académico, $1,6 \%$ (IC95\% 0,2 a 2,7) de quienes informaron excelente rendimiento mostraban consumo diario de cigarrillo; 3,9\% (IC95\% 2,2 a 5,6) de los que manifestaron rendimiento bueno; 4,1\% (IC95\% 2,1 a 6,1 ) de los que consideraron su rendimiento regular, y 9,4\% (IC95\% 3,3 a 15,6) de los que lo anotaron como malo; estas diferencias fueron estadísticamente significativas $(p=0,045)$. Los OR de prevalencia según el rendimiento académico, tomando como referencia el rendimiento excelente, aparecen en el cuadro 1.

En lo referente al consumo de alcohol durante el mes anterior, se encontró que 11,6\% (IC95\% 6,4 a 16,8$)$ de los estudiantes con consumo diario de cigarrillo presentaban riesgo de alcoholismo comparado con 2,8\% (IC95\% 1,8 a 3,7) de los que negaron CDC; esta diferencia fue significativa (OR=2,45; IC95\% 1,64 a 3,76). La prevalencia ajustada de consumo diario de cigarrillo fue 17,6\% (IC95\% 11,1 a 26,7) en los estudiantes con hermanos u otro familiar que consumía alcohol o fumaba versus 2,4\% (IC95\% 1,8 a 3,4) en aquéllos que negaron esta información, diferencia que alcanzó un valor significativo (OR=4,87; IC95\% 1,08 a 2,64). En relación con el consumo de alcohol o cigarrillo por parte del mejor amigo, la prevalencia ajustada de consumo diario de cigarrillo fue de7,9\% (IC95\% 5,8 a 10,9) en quienes lo afirmaron y $1,7 \%$ (IC95\% 1,1 a 2,9) en los que lo negaron, diferencia que fue estadísticamente significativa (OR=5,32; IC95\% 2,48 a 11,49). EI consumo diario de cigarrillo fue independiente de la categoría del colegio, ya que la prevalencia ajustada entre los privados fue de 4,1\% (IC95\% 2,9 a 5,9 ), mientras que en los oficiales fue de $3,7 \%$ (IC95\% 2,8 a 4,9; $p=0,611$ ); tampoco hubo asociación con el estrato socioeconómico, con y sin los datos omitidos $(p=0,401,0,319)$. Las variables que mantuvieron un nivel de significación en el análisis multivariado se muestran en el cuadro 2.

\section{Discusión}

Este estudio informa que el consumo diario de cigarrillo durante el mes anterior se encontraba en $3,8 \%$ de los adolescentes estudiantes de sexto

Cuadro 2. Modelo de regresión logística para consumo diario de cigarrillo en adolescentes estudiantes de básica secundaria de Bucaramanga, Colombia.

\begin{tabular}{lrcc}
\hline Consumo diario de cigarrillo & OR & IC 95\% & $\boldsymbol{p}$ \\
\hline Amigo consume alcohol o fuma & 3,71 & $1,71-8,04$ & 0,001 \\
Edad (tener más años cumplidos) & 1,44 & $1,19-1,75$ & 0,001 \\
Rendimiento académico malo & 3,93 & $1,03-14,18$ & 0,045 \\
Consumo abusivo de alcohol & 2,62 & $0,92-7,42$ & 0,070 \\
Sexo masculino & 1,71 & $0,88-3,31$ & 0,109 \\
\hline
\end{tabular}


a noveno grado. Después de ajustar por otras variables, se observó que tener un mejor amigo fumador o consumidor de alcohol, informar un mal rendimiento académico y tener más años cumplidos de edad se asociaron de forma significativa con consumo diario de cigarrillo.

Sólo algunos estudios más recientes informan la prevalencia de consumo diario de cigarrillo durante el mes anterior en adolescentes. Los datos disponibles varían tanto en función del país como del rango de edad estudiado; por ejemplo en Gran Canaria (España), la prevalencia encontrada en estudiantes de 13 a 14 años (séptimo grado) fue de 6,3\% (32); en estudiantes de Córdoba (España) entre 11 y 17 años de edad (de sexto a noveno grado) fue de $22,6 \%$ (33); en estudiantes de noveno grado de diferentes grupos étnicos en California y Hawai (Estados Unidos) fue de entre 5,8\% y 19,7\% (34); en Florida (Estados Unidos), en estudiantes de 13 a 15 años fue de 9,8\% (35), y en estudiantes de todos los Estados Unidos se informan prevalencias de entre $7,8 \%$ y $18 \%$ $(36,37)$.

En relación con el sexo, en este grupo de edad y escolaridad, los hallazgos son inconsistentes. Un estudio informó que el consumo diario de cigarrillo era mayor en varones que en mujeres (38). Otra investigación encontró que un número significativamente mayor de mujeres que de varones presentaba consumo diario de cigarrillo (32); un dato similar sólo se encontró en mujeres entre 15 y 18 años (35), y un par de investigaciones muestran que el consumo diario de cigarrillo es independiente del género $(33,35)$. Un estudio precisó que esta igualdad únicamente se apreciaba en edades entre 11 y 14 años (36).

En lo concerniente al riesgo de alcoholismo en estudiantes con consumo diario de cigarrillo, los datos discrepan de lo informado por otros autores. Se observa una asociación significativa entre el consumo diario de cigarrillo y un patrón de consumo abusivo de alcohol (32). Es posible que esta diferencia esté relacionada con factores sociales y culturales en este rango de edad. Por otra parte, que el mejor amigo sea fumador se ha relacionado significativamente con consumo diario de cigarrillo (32-34,39). Una asociación similar se ha documentado cuando el contexto familiar, hermano, padres $u$ otro familiar cercano son fumadores $(33,38-40)$. El consumo diario de cigarrillo se ha observado en aquellos estudiantes con resultados académicos menos positivos (35). En relación con la percepción de la propia salud, lo hallado concuerda con un estudio realizado en estudiantes adolescentes de media vocacional de Bucaramanga, en el que no se identificó una relación significativa entre fumar y la percepción del estado de salud (Campo-Arias A, Dallos CM, González-Rueda SJ, Rodríguez DC, SánchezHerrera ZM, Díaz-Martínez LA. Consumo de cigarrillo y síntomas depresivos en estudiantes de Bucaramanga, Colombia. Enviado para publicación).

Para explicar las asociaciones encontradas es importante tener presente que el consumo de cigarrillo es un patrón de conducta con determinantes genéticos y socioculturales $(35,37,41,42)$. Como ejemplo puede anotarse que identificar mayor número de familiares fumadores en aquellos con consumo diario de cigarrillo puede deberse a un factor genético que interactúa con un factor ambiental (imitación). La imitación, igualmente, se propuso para entender la variable de que el mejor amigo sea fumador (43); no obstante, las investigaciones sugieren que la amistad se consolida en la medida en que se compartan características comunes, que generalmente preceden a la relación de amistad (44).

La publicidad juega un papel crucial en el consumo de cigarrillo, pues está orientada principalmente a la población adolescente y aumenta el inicio y la persistencia del consumo (45). Estos resultados sugieren que el consumo diario de cigarrillo se establece en la adolescencia temprana, lo que implica que el consumo de cigarrillo no es una conducta pasajera en un número importante de adolescentes. Igualmente, en adultos se observó que los fumadores intermitentes constituyen un grupo distinto de los que tienen consumo diario de cigarrillo (46). A su vez, el consumo de cigarrillo, junto con el de alcohol, es la puerta de entrada al consumo de sustancias ilegales, y es mucho más probable el consumo de estas sustancias en adolescentes fumadores diarios que 
en fumadores ocasionales (47). Por otro lado, la asociación con un peor rendimiento académico puede ser indicador temprano y objetivo de malestar emocional o trastorno mental, en particular de un trastorno depresivo (48-52). Los trastornos depresivos se incrementan en forma importante después de la pubertad y tienen un impacto desfavorable en la calidad de vida y en el rendimiento académico de los adolescentes estudiantes $(53,54)$.

El inicio y el mantenimiento del consumo de cigarrillo durante la edad escolar o la adolescencia temprana sugieren que las actividades preventivas deben empezar tempranamente. Este proceso debe involucrar a los padres, quienes deben promover la abstinencia y desaconsejar el consumo de cigarrillo en los hijos por medio de un diálogo directo sobre las consecuencias nocivas del consumo y mediante el ejemplo de no consumo de cigarrillo $(55,56)$. De la misma manera, se necesita de la participación de los maestros y profesores en acciones de educación en salud que promuevan conductas saludables, y en la identificación de estados emocionales negativos, especialmente en estudiantes con rendimiento inferior al esperado para su capacidad intelectual (57). Estos estados emocionales pueden precipitar y favorecer el inicio y la consolidación de un patrón de consumo diario de cigarrillo $(50,58)$. Igualmente, los médicos generales, los pediatras, enfermeras y otros profesionales del área de la salud deben aprovechar todas las oportunidades para evaluar la posible condición de fumador de todos los adolescentes que demandan servicios, desalentar el consumo y utilizar todas las estrategias efectivas de que se disponga para incentivar el abandono del hábito en los fumadores (59). Finalmente, para hacer viable este proceso es fundamental la adopción de políticas de salud pública que hagan efectivas las medidas que protegen a los no fumadores, la restricción de la publicidad en algunos lugares y horarios y el aumento de los impuestos a los cigarrillos (60).

Este estudio es el primer informe de consumo diario de cigarrillo en adolescentes estudiantes de básica secundaria en Bucaramanga y en Colombia que toma una muestra representativa de la población escolarizada. Sin embargo, se omitió la identificación de los estudiantes con dependencia de nicotina (no todas las personas que fuman diariamente reúnen criterios para dependencia de nicotina) y la cuantificación de síntomas depresivos (como un indicador del bienestar general) como variable de confusión por carecer hasta la fecha de instrumentos formalmente validados para este propósito en esta población.

Se concluye que en la ciudad de Bucaramanga, de cada 26 estudiantes, aproximadamente uno, cuyo mejor amigo es fumador o consume alcohol y tiene bajo rendimiento académico, presenta consumo diario de cigarrillo. Es necesario determinar la prevalencia de la dependencia a la nicotina en esta población y diseñar programas de prevención para aquellos adolescentes que no han iniciado el consumo, y de promoción del abandono en los que actualmente fuman, con énfasis en quienes muestran consumo diario de cigarrillo.

\section{Conflicto de intereses}

Declaramos no tener ningún conflicto de intereses.

\section{Financiación}

Este trabajo lo financió el Centro de Investigaciones Biomédicas de la Universidad Autónoma de Bucaramanga.

\section{Referencias}

1. Romero PJ, Luna JD, Mora A, Alché V, León MJ. Perfil de los adolescentes de enseñanza secundaria. Estudio comparativo entre el medio rural y urbano. Prev Tab 2000;2:5-16.

2. Jackson C, Dickinson D. Cigarette consumption during childhood and persistence of smoking through adolescence. Arch Pediatr Adolesc Med 2004;158: 1050-6.

3. Ezzatti M, Lopez AD. Estimates of global mortality attributable to smoking in 2000. Lancet 2003;362:84752.

4. Bonard L, Janin-Jacquat B, Michaud PA. Who are the adolescents who stop smoking? Eur J Pediatr 2001; 160:430-5.

5. Katja R, Päivi A-K, Marja-Terttu T, Pekka L. Relationship among adolescent subjective well-being, health behavior, and school satisfaction. J Sch Health 2002;72:243-9. 
6. Mokdad AH, Marks JS, Stroup DF, Gerberding JL. Actual causes of death in the United States, 2000. JAMA 2004;291:1238-45.

7. Terazón $\mathbf{0}$, Sánchez $\mathbf{N}$. Adolescencia y hábito de fumar. Medisan 1998;2:47-50.

8. Simantov E, Schoen C, Klein JD. Healthcompromising behaviors: Why do adolescents smoke or drink? Arch Pediatr Adolesc Med 2000;154:1025-33.

9. Padgett DI, Selwyn BJ, Kelder SH. Ecuadorian adolescents and cigarette smoking: a cross-sectional survey. Rev Panam Salud Pública 1998;4:87-93.

10. Ariza C, Nebot M, Villalbí JR, Díez E, Tomás Z, Valmayor S. Tendencias en el consumo de tabaco, alcohol y cannabis de los escolares de Barcelona (1987-1999). Gac Sanit 2003;17:190-5.

11. Azevedo A, Machado AP, Barros H. Tobacco smoking among Portuguese high-school students. Bull World Health Organ 1999;77:509-14.

12. Pérez MA, Pinzón-Pérez H. Alcohol, tobacco, and other psychoactive drug use among high school students in Bogota, Colombia. J Sch Health 2000;70: 377-80.

13. Everett SA, Malarcher AM, Sharp DJ, Husten CG, Giovino GA. Relationship between cigarette, smokeless tobacco, and cigar use, and other health risk behaviors among US high school students. J Sch Health 2000;70:234-40.

14. Rueda GE. Diagnóstico del consumo de sustancias psicoactivas en los estudiantes de enseñanza media vocacional. Bucaramanga: Grafisur; 1997. p.1-19.

15. Rueda GE, Camacho PA. Vigilancia epidemiológica sobre el uso indebido de sustancias psicoactivas en los estudiantes de enseñanza media vocacional de Bucaramanga y su área metropolitana. Médicas UIS 1998;12:107-13.

16. República de Colombia. Ley 84 de 1989.

17. Ministerio de Salud de Colombia. Resolución 008430 por la cual se establecen las normas científicas, técnicas y administrativas para la investigación en salud, 1993.

18. Restrepo B, Restrepo G, Acevedo J. VESPA en décimo y undécimo de enseñanza media vocacional. 2 ed. Medellín: Fundación Luis Amigó; 1994. p.183-97.

19. Torres de Galvis Y. Sistema de vigilancia epidemiológica sobre uso indebido de sustancias psicoactivas. Medellín: Martín Vieco; 1994. p.1-85.

20. American Psychiatric Association. Diagnostic and statistical manual of mental disorders. Fourth Edition. Washington, D.C.: American Psychiatric Association; 1994.

21. Organización Mundial de la Salud. Clasificación internacional de las enfermedades (CIE). Trastornos mentales y del comportamiento. Criterios diagnósticos de investigación.10a edición. Madrid: Meditor; 1993.

22. Breslau N, Novak SP, Kessler RC. Daily smoking and the subsequent onset of psychiatric disorders. Psychol Med 2004;34:323-33.

23. Ewing JA. Detecting alcoholism. The CAGE questionnaire. JAMA 1984;252:1905-7.

24. Ewing JA. CAGE questionnaire. En: Rush AJ, Pincus $\mathrm{HA}$, First MB, Zarin DA, Blacker D, Endicott $\mathrm{J}$ et al, editors. Handbook of psychiatric measures. Washington, D.C.: American Psychiatric Association; 2002. (CD-ROM).

25. Kuder GF, Richardson MW. The theory of the estimation of test reliability. Psychometrika 1937;2:15160.

26. Cronbach LJ. Coefficient alpha and the internal structure of test. Psychometrika 1951;16:297-334.

27. Blacker D, Endicott J. Psychometric properties: concepts of reliability and validity. En: Rush AJ, Pincus $\mathrm{HA}$, First MB, Zarin DA, Blacker D, Endicott $\mathrm{J}$ et al., editors. Handbook of psychiatric measures. Washington, D.C.: American Psychiatric Association; 2002 (CD-ROM).

28. STATA 9.0 for Windows 12.0. College Station, StataCorp LP, 2005.

29. STATA. Svy: tabulate twoway - Two-way tables for survey data. En: Stata survey data. Reference manual. Release 9. College Station: Stata Press; 2005. p.21230.

30. Hernández B, Velasco-Mondragón HE. Encuestas transversales. Salud Pública Mex 2000;42:447-55.

31. Greenland S. Modeling and variable selection in epidemiologic analysis. Am J Public Health 1989;79: 340-9.

32. Pinilla J, González B, Barber P, Santana Y. Smoking in young adolescents: an approach with multilevel discrete choice models. J Epidemiol Community Health 2002;56:227-32.

33. Ortiz S, Peña D, Ortiz S, Sánchez R. Consumo de tabaco de los estudiantes de secundaria de Córdoba. Enferm Clínica 2003;13:154-8.

34. Wong MM, Klingle RS, Price RK. Alcohol, tobacco, and other drug use among Asian American and Pacific Islander in California and Hawaii. Addict Behav 2004; 29:127-41.

35. Zapata LB, Forthofer MS, Eaton DK, Brown KM, Bryant CA, Reynolds ST et al. Cigarette use in 6th through 10th grade: The Sarasota County Demonstration Projet. Am J Health Behav 2004;28:151-65.

36. Mowery PD, Farrelly MC, Haviland L, Gable JM, Wells HE. Progression to established smoking among US youth. Am J Public Health 2004;94:331-7. 
37. van der Bree MB, Whitmer MD, Pickworth WB. Predictors of smoking development in a populationbased sample of adolescents: a prospective study. J Adolesc Health 2004;35:172-81.

38. Eckhardt L, Woodruff SI, Elder JP. A longitudinal analysis of adolescent smoking and its correlates. J Sch Health 1994;64:67-72.

39. Poikolainen K, Tulio-Henriksson A, Aalto-Setälä T, Marttunen M, Lönnqvist J. Predictors of alcohol intake and heavy drinking in early adulthood: a 5-year followup of 15-19-year-old Finnish adolescents. Alcohol 2001; 36:85-8.

40. Johnson CC, Li D, Perry CL, Elder JP, Feldman HA, Kelder SH et al. Fifth through eighth grade longitudinal predictors of tobacco use among a racially diverse cohort: CATCH. J Sch Health 2002;72:58-64

41. Poulsen LH, Osler M, Roberts C, Due P, Damsgaard MT, Holstein BE. Exposure to teachers smoking and adolescent smoking behaviour: analysis of cross sectional data from Denmark. Tob Control 2002;11: 246-51.

42. Rhee SH, Hewitt JK, Young SE, Corley RP, Crowley TJ, Stalling MC. Genetic and environmental influences on substance initiation, use, and problem use in adolescents. Arch Gen Psychiatry 2003;60:1256-64.

43. Pombeni ML, Kirchler E, Palmonari A. Identification with peers as a strategy to muddle through the troubles of the adolescent years. J Adolesc 1990;13:351-69.

44. Michell L, West P. Peer pressure to smoke: the meaning depends on methods. Health Educ Res 1996;11:39-49.

45. Braverman MT, Aaro LE. Adolescent smoking and exposure to tobacco marketing under a tobacco advertising ban: findings from 2 Norwegian national samples. Am J Public Health 2004;94:1230-8.

46. Pierce JP, García M, Saltó E, Schiaffino A, Fernández E. La publicidad del tabaco ¿incrementa el hábito de fumar entre los adolescentes? Datos desde California. Gac Sanit 2002;16:69-76.

47. Lewinsohn PM, Rohde P, Brown RA. Level of current and past adolescent cigarette smoking as predictor of future substance use disorders in young adulthood. Addiction 1999;94:913-21.

48. Brown RA, Lewinsohn PM, Seeley JR, Wagner EF. Cigarette smoking, major depression, and other psychiatric disorders among adolescents. J Am Acad Child Adolesc Psychiatry 1996;35:1602-10.

49. Escobedo LG, Reddy M, Giovino GA. The relationship between depressive symptoms and cigarette smoking in US adolescents. Addiction 1998; 93:433-40.

50. Patton GC, Carlin JB, Coffey C, Wolfe R, Hibbert $\mathbf{M}$, Bowes $\mathbf{G}$. Depression, anxiety, and smoking initiation: a prospective study over 3 years. J Am Public Health 1998;88:1518-22.

51. McGee R, Williams S, Poulton R, Moffitt T. A longitudinal study of cannabis use and mental health from adolescence to early adulthood. Addiction 2000; 95:491-503.

52. Upadhyaya HP, Deas D, Brady KT, Kruesi M. Cigarette smoking and psychiatric comorbidity in children and adolescents. J Am Acad Child Adolesc Psychiatry 2002;41:1294-305.

53. Field T, Diego M, Sanders C. Adolescent depression and risk factor. Adolescence 2001;36:491-8.

54. Hesketh T, Ding QJ, Jenkins R. Suicide ideation in Chinese adolescents. Soc Psychiatry Psychiatr Epidemiol 2002;37:230-5.

55. Chassin L, Presson C, Rose J, Sherman SJ, Prost J. Parental smoking cessation and adolescent smoking. J Pediatr Psychol 2002;27:485-96.

56. Kold MM, Mermelstein R. Beyond modeling: parenting practices, parental smoking history, and adolescent cigarette smoking. Addict Behav 2004;29:17-32.

57. Martin MW, Levins S, Saunders R. Secondary school tobacco policy and prevention curricula in South Carolina. Nicotine Tob Res 1999;1:341-6.

58. Vogel JS, Hurford DP, Smith JV, Cole AK. The relationship between depression and smoking in adolescents. Adolescence 2003;38:57-74.

59. Benuck I, Gidding SS, Binns HJ. Identification of adolescent tobacco use in a pediatric practice. Arch Pediatr Adolesc Med 2001;155:32-5.

60. Wakefield MA, Chaloupka FJ, Kaufman NJ, Orleans CT, Barker DC, Ruel EE. Effect of restrictions on smoking at home, at school, and in public places on teenage smoking: cross sectional study. BMJ 2000; 321:333-7. 